\title{
Effects of morphology on phonons in nanoscopic silver grains
}

\author{
Gustavo A. Narvaez, ${ }^{1, *}$ Jeongnim Kim, ${ }^{2,3}$ and John W. Wilkins ${ }^{1}$ \\ ${ }^{1}$ Department of Physics, The Ohio State University, Columbus, Ohio 43210, USA \\ ${ }^{2}$ Materials Computation Center, University of Illinois, Urbana-Champaign, Illinois 61801, USA \\ ${ }^{3}$ National Center for Supercomputer Applications, University of Illinois, Urbana-Champaign, Illinois 61801, USA \\ (Received 18 November 2004; revised manuscript received 15 August 2005; published 14 October 2005)
}

\begin{abstract}
The morphology of nanoscopic Ag grains significantly affects the phonons. Atomistic simulations show that realistic nanograin models display complex vibrational properties. (1) Single-crystalline grains. Nearly pure torsional and radial phonons appear at low frequencies. For low-energy, faceted models, the breathing mode and acoustic gap (lowest frequency) are about $10 \%$ lower than predicted by elasticity theory (ET) for a continuum sphere of the same volume. The sharp edges and the atomic lattice split the ET-acoustic-gap quintet into a doublet and triplet. The surface protrusions associated with nearly spherical, high-energy models produce a smaller acoustic gap and a higher vibrational density of states (DOS) at frequencies $\nu<2 \mathrm{THz}$. (2) Twined icosahedra. In contrast to the single-crystal case, the inherent strain produce a larger acoustic gap, while the core atoms yield a DOS tail extending beyond the highest frequency of single-crystalline grains. (3) Mark's decahedra, in contrast to (1) and (2), do not have a breathing mode; although twined and strained, do not exhibit a high-frequency tail in the DOS. (4) Irregular nanograins. Grain boundaries and surface disorder yield nondegenerate phonon frequencies, and significantly smaller acoustic gap. Only these nanograins exhibit a low-frequency $\nu^{2}$ DOS in the interval 1-2 THz.
\end{abstract}

DOI: 10.1103/PhysRevB.72.155411

PACS number(s): 63.22.+m, 78.67.Bf

\section{INTRODUCTION}

Recent experiments have probed the size dependence of the electronic-energy relaxation rate in nanoscopic metal grains. ${ }^{1-3}$ Perhaps the most striking aspect of these experiments is the finding of Arbouet and coworkers which shows that the electron-phonon relaxation rate $\tau_{\text {ep }}$ does not depend on the environment of the grains. ${ }^{1}$ Gold and silver grains suspended in solution present the same size dependence of $\tau_{\text {ep }}$ than those embedded in a solid matrix (e.g., $\mathrm{Al}_{2} \mathrm{O}_{3}$ ).

To explain the data, current models for the energy relaxation of hot electrons due to electron-phonon coupling in nanoscopic grains rely on phenomenological parameters and simplifications. (For example, replacing the vibrational density of states with the Debye model.) One must keep in mind, however, that the experiments involved several materials $(\mathrm{Ag}, \mathrm{Au}, \mathrm{Pt})$ with a wide range of grain sizes, where a myriad of stable low-energy morphologies has been observed and proposed. ${ }^{4-7}$ Thus, to understand the electron-phonon coupling in nanoscopic metal grains it would be desirable to have a description of their vibrational properties that includes the grain's morphology: facets, grain boundaries, and surface disorder and defects.

There are papers in the literature that have addressed the vibrational density of states of nanoscopic metal grains using ideal, spherical models. ${ }^{8-10}$ Unfortunately, the use of these idealized models limits a detailed understanding of the vibrational properties and the role of morphology. Furthermore, the idealized models neglect the influence of grain boundaries, and surface disorder and defects on the vibrational properties of the experimental nanograins. ${ }^{11}$ In this work we focus on realistic, nanoscopic Ag grains containing 1501400 atoms and simulate atomistically their vibrational properties, by calculating the phonon frequencies and displacement vectors. We show that morphology introduces a high degree of complexity in the phonon spectra; total and partial vibrational density of states; and phonon localization.

Our predictions, which should be general for nanoscopic metals, show the following prominent features. (a) Lowenergy, single-crystalline grains present nearly pure torsional and radial phonon modes. The frequencies of the breathing mode and the acoustic gap (lowest frequency) are nearly $10 \%$ lower than predicted by elasticity theory (ET) for a continuum sphere. ${ }^{12-14}$ The sharp edges and atomic lattice of the grains lead to the splitting of the acoustic gap quintet predicted by ET in a doublet and triplet, where the magnitude of the splitting depends on the relative number of atoms at the boundary of different facets. High-energy, ideal spherical models present regular protrusions on the surface. When compared to a faceted grain of the same size, these protrusions lead to a smaller acoustic gap, which is not necessarily degenerate; and to a higher total vibrational density of states (DOS) at frequencies $\nu<2 \mathrm{THz}$.

(b) As in the case of single-crystalline nanograins, twined icosahedra have a breathing mode. Strain in these grains leads to a higher acoustic gap, and a high-frequency tail in the DOS that extends beyond the highest frequency in singlecrystalline grains. On the other hand, twined and strained Mark's decahedra do not have a breathing mode, neither exhibit a high-frequency tail on DOS.

(c) Irregular nanograins with grain boundaries and surface disorder do not have degenerate phonon frequencies, and the acoustic gap is significantly reduced. These nanograins are the only ones to exhibit $\nu^{2}$ DOS in the interval 1-2 THz. The extent of this region depends on the nature of disorder.

To summarize, our simulations show the complexity of the vibrational properties of nanoscopic metals, and point out similarities between the vibrational density of states of nanograins with grain boundaries and surface disorder and that of massive nanocrystalline samples. This work is a required 
a)

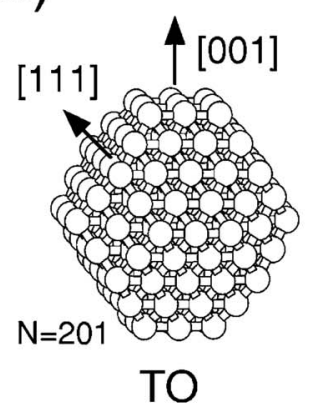

Class I

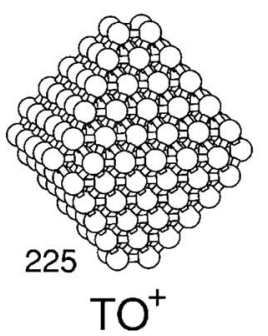

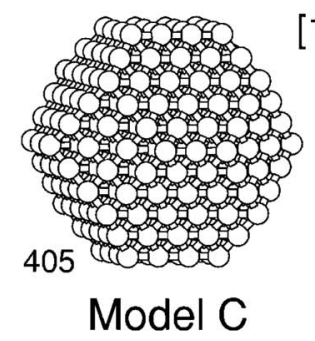

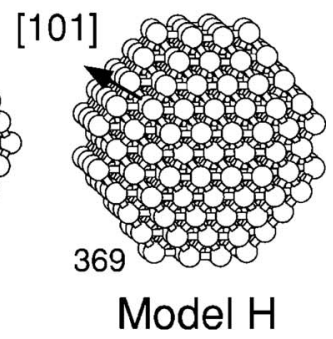

FIG. 1. Classes of $\mathrm{Ag}$ nanograins illustrated with selected specimens. " 5 " $[(\mathrm{b})]$ and " 5 ", $[(\mathrm{c})]$ indicate, respectively, a fivefold and fivefold-like axis. Number of atoms $(N)$ is indicated. (a) Single-crystal grains with [111] and [001] facets in the regular (TO) and irregular $\left(\mathrm{TO}^{+}\right)$truncated octahedron geometry. Compared to $\mathrm{TO}^{+}$, Model $\mathrm{C}$ has bigger [001] facets and similar [111]facet geometry. Model $\mathrm{H}$ grains present [101] facets. (b) Twined icosahedron (Ih) and Mark's decahedron (M-Dh). (c) Multigrain nanograins exhibit grain boundaries and surface disorder; gray atoms highlight vertex vacancies. Spherical nanograins result from decorating regular, faceted specimens. Gray atoms decorating [111] facets of TO [see (a)] yield the shown "sphere."

step towards understanding the size dependence of the electron-phonon coupling in nanoscopic metal grains.

\section{NANOGRAINS' MORPHOLOGY AND ENERGETICS}

The morphology of a nanograin is specified by the number of atoms $N$, shape and atomic arrangement. The shape refers to the symmetry and facets, while the atomic arrangement pertains to the nanograin's crystalline characteristics: single-crystalline, twined, surface-disordered, and defective. Here, we classify the model nanograins-with sizes that range from $N=150-1400$ - in three broad classes:

(I) Single-crystalline nanograins derived from the facecentered-cubic lattice of Ag with facets oriented in the [111], [001], and [101] directions (depending on size). Figure 1(a) illustrates class I grains. $\mathrm{TO}$ and $\mathrm{TO}^{+}$originate from different degrees of truncation of an octahedron $(\mathrm{Oh})$. TOs are regular in the sense that the number of atoms in every edge is the same and the [111] and [001] facets form regular hexagons and squares, respectively. $\mathrm{TO}^{+}$represents a group of grains that result from removing the vertices of the Oh first and then [001] facets until reaching the TO. The latter protocol leads to grains that have more atoms in the edges connecting [111] facets than in the $[111] /[001]$ boundary. Model $\mathrm{C}$ results from truncating cuboctahedra. In these grains, [001] facets are larger than the corresponding facets in $\mathrm{TO}^{+}$, while the geometry of [111] facets is preserved. On the other hand, by simply removing the atoms lying in the [111]-facets boundary, Model C leads to Model H. By construction, Model H nanograins show [101] facets.

(II) Twined icosahedra (Ih) and its variants, ${ }^{15}$ and Mark's decahedra (M-Dh). Examples of Ih and M-Dh appear in Fig. 1. Both these grains are described in detail in the literature. ${ }^{16,17}$ Ih's are strained and feature [111] facets, 12 vertices, 20 internal grains, and six fivefold axis; one of them appears indicated with a " 5 " in Fig. 1. In contrast, M-Dh's are less strained, have multiple faceting and a single fivefold axis.

(III) Complex, partially disordered multigrain nanograins that have grain boundaries, surface disorder, and defects; see Fig. 1(c). Simulated annealing is used to generate these grains. The most common surface defects are vertex vacancies [shown in grey in Fig. 1(c)], terraces, and stacking faults. In most cases, the grain boundaries lead to fivefoldlike axis and a high number (about 10) of internal grains. However, we have also encountered multigrains with a single twin boundary or internal stacking fault.

We also include in class III the so-called spherical nanograins [Fig. 1(c)]. Despite their unrealistic morphology, these grains have been the model of choice in recent simulations of the vibrational properties of nanoscopic metal grains. ${ }^{8,10}$ Although single-crystalline, these grains have small protrusions (grey atoms in the figure) on the [111] and, depending on size, [001] facets of otherwise compact specimens. These protrusions act as surface defects, despite being highly regular. (Indeed, one may consider the compact nanograins to be decorated by the protrusions.) These surface defects contribute to the higher energies of the spherical nanograins relative to other structures and are removed by simulated annealing starting at high temperatures.

Figure 2 shows the effect of morphology on the total energy $\mathcal{E}$ for classes I and II. Ih are the most stable grains up to $N \simeq 600$, where the cohesive energies of $\mathrm{Ih}, \mathrm{M}-\mathrm{Dh}$, and TO are effectively degenerate. For larger grains, strain dominates over efficient faceting and single-crystalline grains become more stable. $\mathrm{TO}^{+}$splits into two bands, indexed by the number of atoms in [111]-facet boundaries; the lower and upper bands corresponding to 5 and 7 atoms, respectively. The lower and upper band merge at $N=1289$ and $N=1975$, re- 


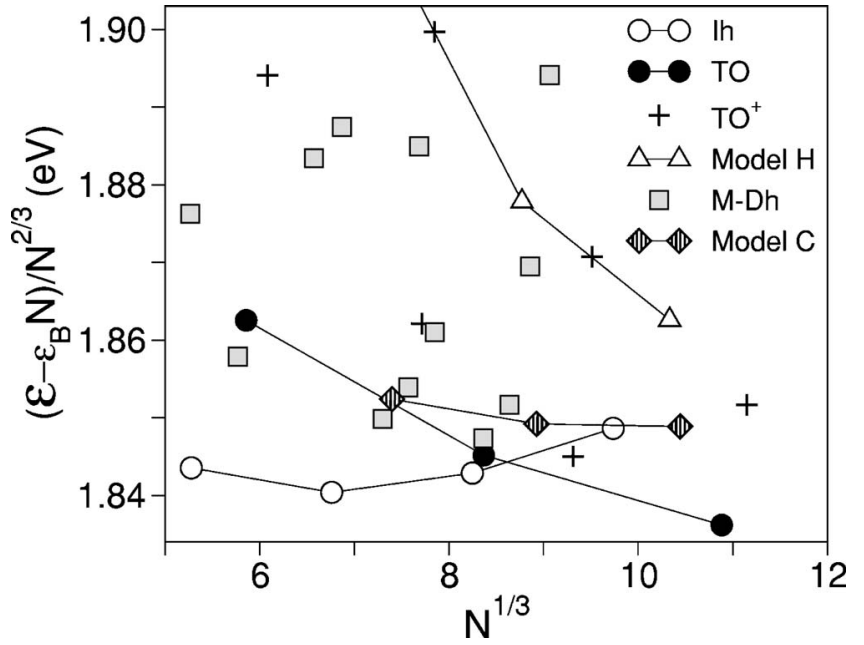

FIG. 2. Morphology effects on the total energy $\mathcal{E}$ of grains in Figs. 1(a) and 1(b). $\mathcal{E}$ is measured relative to bulk $\left(\varepsilon_{B}=2.85 \mathrm{eV}\right)$. Twined icosahedra (Ih) are the most stable structures from 150 to $N \simeq 600$; where the cohesive energies of $\mathrm{Ih}, \mathrm{M}-\mathrm{Dh}$, and TO are nearly degenerate. The energy of $\mathrm{TO}^{+}$nanograins separates in two bands (see Sec. II). The upper band follows the progression of Model $\mathrm{H}$. Increased faceting is responsible for the noticeable scattering in M-Dh's energy.

spectively, with $\mathrm{TO}$ energies. Model $\mathrm{H}$ tracks the $\mathrm{TO}^{+}$upper band. Mark's decahedra show significant fluctuations in the size dependence of $\mathcal{E}$ due to increased faceting; which permits generating $\mathrm{M}$-Dh grains with significantly different sphericity even if the number of atoms is slightly changed. ${ }^{18}$

Considering the energetics of class III, Table I lists the total energies of multigrain and spherical nanograins. These energies exceed by a considerable amount those of classes I and II. Nonetheless, multigrain nanograins are nonequilibrium structures that are likely to exist under experimental conditions. In addition, the recent simulations of the structure of ultrasmall Al clusters (Manninen et al., in Ref. 18) show that complex, multigrain structures are competitive with those of classes I and II. Spherical grains, on the other hand, correspond to an idealization of realistic metal grains and are presented here only for comparison purposes.

\section{VIBRATIONAL PROPERTIES}

We show that nanograin morphology strongly affects the vibrational properties. By using the methods described in the Appendix, we analyze the effect of nanograin's morphology on the phonon frequency spectrum, vibrational density of

TABLE I. Total energy of multigrain and spherical Ag grains vs number of atoms $N$. Here, $\varepsilon_{B}=2.85 \mathrm{eV}$ is the bulk cohesive energy.

\begin{tabular}{ccccc}
\hline \hline & \multicolumn{4}{c}{$\left(\mathcal{E}-\varepsilon_{B} N\right) / N^{2 / 3}(\mathrm{eV})$} \\
\cline { 2 - 5 }$N$ & 225 & 459 & 783 & 1289 \\
\hline Multigrain & 1.93 & 1.94 & 1.98 & \\
Spherical & 2.06 & 2.06 & 1.99 & 2.04 \\
\hline \hline
\end{tabular}

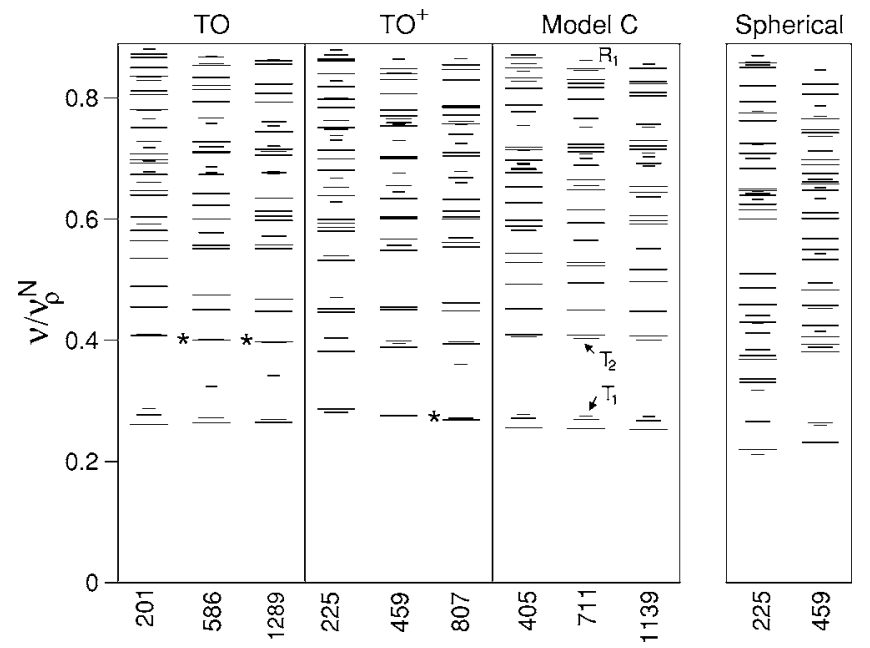

FIG. 3. Single-crystalline Ag grains' scaled phonon frequencies $\nu$ up to the first nearly radial, breathing mode $\left(R_{1}\right) . \nu_{\rho}^{N}$ $=2.825\left(c_{l} / 2 \pi R\right)$ (see Sec. III A). Frequencies are either nondegenerate (short-dashed line) or twofold (medium dashed line) and threefold (long dashed line) degenerate. In $\mathrm{TO}, \mathrm{TO}^{+}$and Model $\mathrm{C}$, the low-lying phonon frequency structure is qualitatively similar except for $T_{1}$; a nearly torsional, edge vibration (see Fig. 4). $T_{2}$ labels a twofold degenerate twist mode. Asterisks $\left({ }^{*}\right)$ indicate nearly degenerate doublet-triplet clusters. Surface protrusions yield different low-frequency phonon structure in spherical grains.

states (DOS), and phonon localization. First, we describe general features of phonon spectra followed by a comparison between class I and spherical grains with class II and multigrains. Second, we contrast the DOS of TO with classes II and III nanograins. Finally, we focus on $N=459$ nanograins in classes I and III to illustrate important features of the phonon localization.

\section{A. Phonon frequencies}

General features: The finite size of the grains lead to discrete phonon spectra. The acoustic gap, which is defined as the value of the lowest phonon frequency, decreases with size due to confinement. Our simulations show that the acoustic gap roughly scales as $N^{-1 / 3}$, as predicted by elasticity theory (ET) for a finite grain, ${ }^{12}$ and its magnitude depends on morphology. Each grain in class I as well as icosahedra (Ih) have a nearly radial, breathing mode. In contrast, this mode does not appear in Mark's decahedra (M-Dh) or multigrains (class III). When compared with predictions of elasticity theory for a solid sphere with the same volume as the nanograins, our simulations lead to frequencies of the breathing mode $\left(R_{1}\right)$ and acoustic gap about $10 \%$ smaller. For $R_{1}$, ET predicts $\nu_{\rho}^{N}=2.825\left(c_{l} / 2 \pi R\right)$, where $R$ is the radius of the sphere and $c_{l}=3686 \mathrm{~m} / \mathrm{s}$ is the average longitudinal sound velocity of bulk silver. ${ }^{19}$ The ET-acoustic-gap equals $0.401 \nu_{\rho}^{N}$ with fivefold degeneracy. ${ }^{13,14}$

Class I and spherical nanograins: For $\mathrm{TO}, \mathrm{TO}^{+}$, and Model C in class I, and for the spherical grains in class III, Fig. 3 shows phonon frequencies up to the first nearly radial, breathing mode. Main features are the following. (i) All grains show frequencies that are either nondegenerate or 

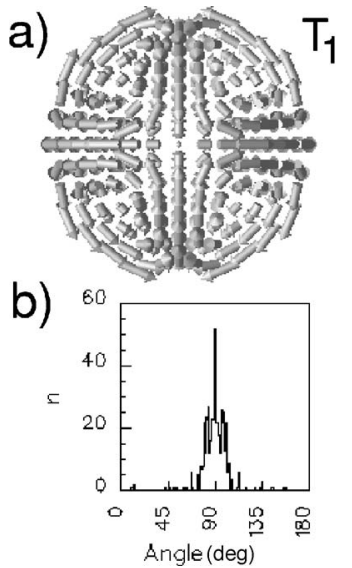
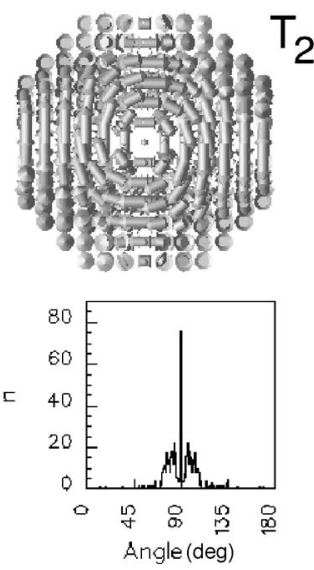
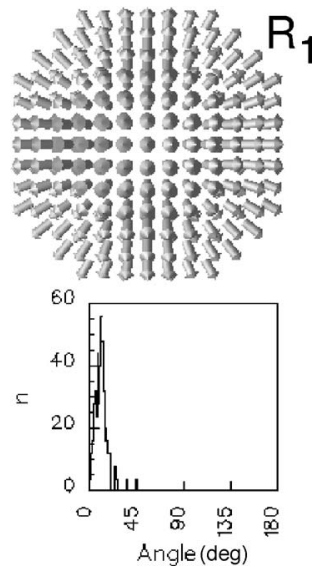

FIG. 4. (a) Displacement vectors of selected phonon modes in the $N=586$, TO nanograin: (i) $T_{1}$, a nondegenerate mode in which the atoms surrounding the edges have a significant amplitude, (ii) $T_{2}$, one of the doubly degenerate twist modes, and (iii) $R_{1}$, the first nearly radial, breathing mode. (b) Distribution functions $\left(1^{\circ}\right.$ bin size $)$ of the angle between the equilibrium vector position of an atom-measured from the center of the grain-and its displacement vector. The distributions peak around $90^{\circ}$ for $T_{1}$ and $T_{2}$, and $10^{\circ}$ (instead zero) for $R_{1}$, respectively. For larger grains the distribution for $R_{1}$ peaks around $2^{\circ}-4^{\circ}$. twofold and threefold degenerate. (ii) The five lowest frequencies split into a triplet and a doublet; a gap separates this cluster from other frequencies. This triplet-doublet cluster originates from symmetry breaking of the ET-acoustic-gap quintet. The size dependence of the small split-off separation between the triplet and doublet depends on the morphology. It decreases for $\mathrm{TO}$, and in $\mathrm{TO}^{+}$with $N=459$ the triplet and doublet even switch order. Model $\mathrm{C}$ shows a more remarkable size dependence: The split-off separation remains nearly constant and similar to that of the TO grain with $N=201$. We attribute this behavior to the fixed number of atoms (3) in the [111]-facet boundaries. (iii) In $\mathrm{TO}^{+}$and in 586 and $1289 \mathrm{TO}$ grains, a distinguishable, second group of frequencies appears; a triplet precedes a doublet and two triplets follow. The separation between multiplets in these complexes also depend upon the size of the nanograin. In contrast, no visible gap separates these frequencies in Model C and TO with $N$ $=201$. At frequencies higher than $0.5 \nu_{\rho}^{N}$ no sizeable gaps appear in any grain. (iv) In all grains, near to the lowest triplet-doublet, we find a low-frequency nearly torsional edge mode $T_{1}$; morphology dramatically affects the size dependence of its frequency (Fig. 3). In TO, $T_{1}$ 's frequency remains almost constant while in $\mathrm{TO}^{+}$it decreases with the surface. In Model $\mathrm{C}$, it scales as $\nu_{\rho}^{N}$ and is locked with the lowest five frequencies.

The ideal spherical nanograins present phonon frequencies that are nondegenerate as well as twofold and threefold degenerate. Contrary to class I grains, the low-lying frequencies present a less clear pattern. These grains, $N=225$ and 459 , may be viewed as the decorated version of the TO with $N=201$ (cf. Fig. 1) and the $\mathrm{TO}^{+}$with $N=405$, respectively. The coupling between the vibration of the protrusions and the atoms in the faceted grain that they decorate reduces the acoustic gap. This coupling also destroys the similarities between the frequency structure in 201 and 405. (See Fig. 3.)

For the TO nanograin with $N=586$, Fig. 4(a) shows representations of the displacement vectors of three selected phonon modes. As mentioned above, $T_{1}$ is nearly torsional and the atoms surrounding the edges have the higher amplitude. $T_{2}$ is one of the two nearly torsional, twofold generate twist modes. In contrast to $T_{1}, T_{2}$ 's frequency depends less strongly on morphology (see Fig. 3). $T_{1}$ and $T_{2}$ also appear in spherical grains. $R_{1}$ is the nondegenerate, nearly radial breathing mode. It should be noted that TO and Ih grains have another nearly torsional mode $T_{3}$ (not shown), which is an excited, nondegenerate twist mode. This mode does not appear in any other single-crystalline grain or Mark decahedron, as only TO's and Ih's have the same number of atoms at the every [111]-facet boundary [cf. Figs. 1(a) and 1(b)].

For $T_{1}, T_{2}, R_{1}$, Fig. 4(b) shows histograms of the angles between the displacement vector of atom $i\left(\vec{u}^{i}\right)$ and its equilibrium position $\left(\vec{R}^{i}\right)$; measured from the center of the grain. [The angle is defined $\bmod (\pi)$.] The purpose is to show the extent to which the crystalline field and edges of the grains couple angular and radial degree's of freedom. Note that in an ideal elastic sphere, torsional and breathing modes have angular- and radial-only displacements, respectively. ${ }^{13}$ The values for the average angle $\bar{\theta}$ and the standard deviation $\sigma$ of the histograms in Fig. 4 are the following: $\bar{\theta}_{T_{1}}=89.51^{\circ}$, $\sigma_{T_{1}}=12.53^{\circ} ; \bar{\theta}_{T_{2}}=89.95^{\circ}, \sigma_{T_{2}}=14.46^{\circ} ;$ and $\bar{\theta}_{R_{1}}=10.64^{\circ}, \sigma_{R_{1}}$ $=5.96^{\circ}$. In general, as size increases, for nearly torsional and radial modes, $\bar{\theta}$ approaches $90^{\circ}$ and $0^{\circ}$, respectively; and $\sigma$ decreases.

Class II and multigrain nanograins: Figure 5(a) shows the phonon frequencies up to the first nearly radial, breathing mode of twined icosahedra (Ih). Panel (b) shows phonon frequencies up to $\nu_{\rho}^{N}$ for Mark's decahedra (M-Dh) and multigrain samples. (See Fig. 1.) Prominent features are the following.

(i) Strain in the icosahedra leads to clusters of nondegenerate frequencies. At higher frequencies a few twofold degeneracies appear. The acoustic gap is higher than in class I and spherical grains. It also has a triplet-doublet structure with higher split-off and no degeneracies. (ii) Mark's decahedra, like Ih's, show only a few doubly degenerate frequencies over the entire spectrum. The acoustic gap is similar in magnitude to the gaps of class I and spherical grains. However, there is no resemblance of the triplet-doublet structure. More importantly, Mark's decahedra do not have breathing modes. The small [111]-oriented dents (see Fig. 1) may be responsible for these features. (iii) Multigrains only have nondegenerate frequencies. Like $\mathrm{M}-\mathrm{Dh}$, multigrain do not have breathing modes. Surface disorder and defects reduce the acoustic gap. (Compare Figs. 3 and 4.) The lowest-lying modes are localized on the surface of the grain; however, the degree of localization is sample (disorder) dependent. In addition, the more localized the mode the softer its frequency. 
a)

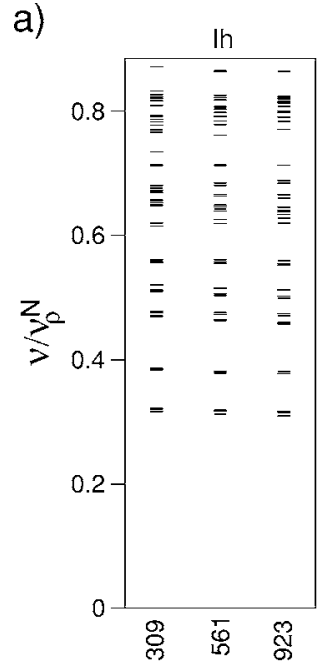

b)

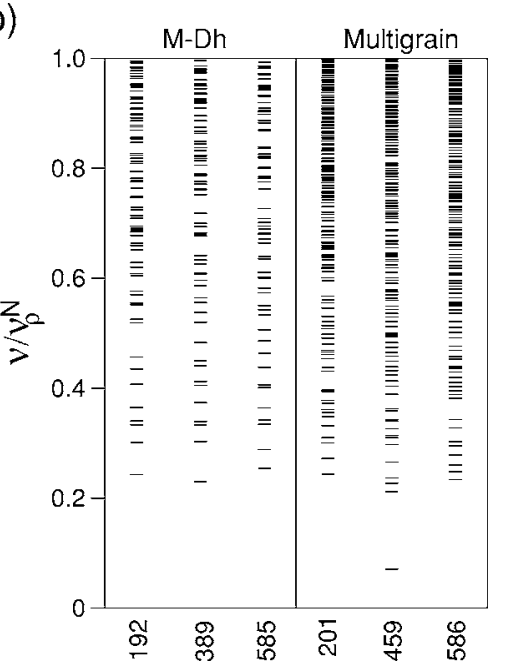

FIG. 5. (a) Equivalent to Fig. 3 but for twined icosahedral grains. Strain leads to clusters of nondegenerate frequencies, and acoustic gaps that are bigger than in single-crystalline grains and Mark's decahedra. (b) Mark's decahedra and multigrain's phonon frequencies up to $\nu_{\rho}^{N}$. These two nanograins do not have nearly radial phonon modes. Like Ih's, M-Dh's show nondegenerate frequencies in this range. In multigrain, grain boundaries and surface disorder lead to nondegenerate frequencies and significantly smaller acoustic gap.

The visible gap between the lowest allowed phonon mode and the first excited vibration in the $N=459$ nanograin is disorder sensitive.

\section{B. Vibrational density of states}

Figure 6 shows the effects of morphology and size on the vibrational density of states (DOS) per atom $g(\nu)$. In these results, each $g(\nu)$ histogram has a $0.1 \mathrm{THz}$ bin. ${ }^{20}$ At low and high frequencies, $g(\nu)$ depends strongly on TO grains size. DOS in TO grains increase up to $2 \mathrm{THz}$, which correspond to a maximum in bulk DOS, originated by transversal phonons (TP). The longitudinal-phonon (LP) peaks around $4.7 \mathrm{THz}$ have nearly the same amplitude for different sizes. Other class I grains (cf. Sec. II) show a similar behavior. DOS extends to slightly higher frequencies for smaller grains. Icosahedra and Mark's decahedra: In icosahedra, strain and twining remarkably smear the LP peak in the DOS. Its intensity is lower than the TP peak about $2 \mathrm{THz}$, and remains roughly unchanged with increasing size. In addition, there is a frequency tail beyond the highest frequency in TO and spherical grains. Beyond $6 \mathrm{THz}$, the DOS show small features (arrows in Fig. 6, Ih) that originate in four phonon modes strongly localized at the center of the grains. These phonon modes consist of displacement of a few (5-40) atoms along the grain-boundary interfaces that meet at the center of grain. Removal of the center atom of the icosahedra results in the disappearance of these features in the density of states. Mark's decahedra show a DOS significantly different from icosahedra; see the inset in Fig. 6, panel Ih, 561. Although M-Dh's are twined and strained, an LP peak is visible and its intensity remains nearly unchanged with size (not
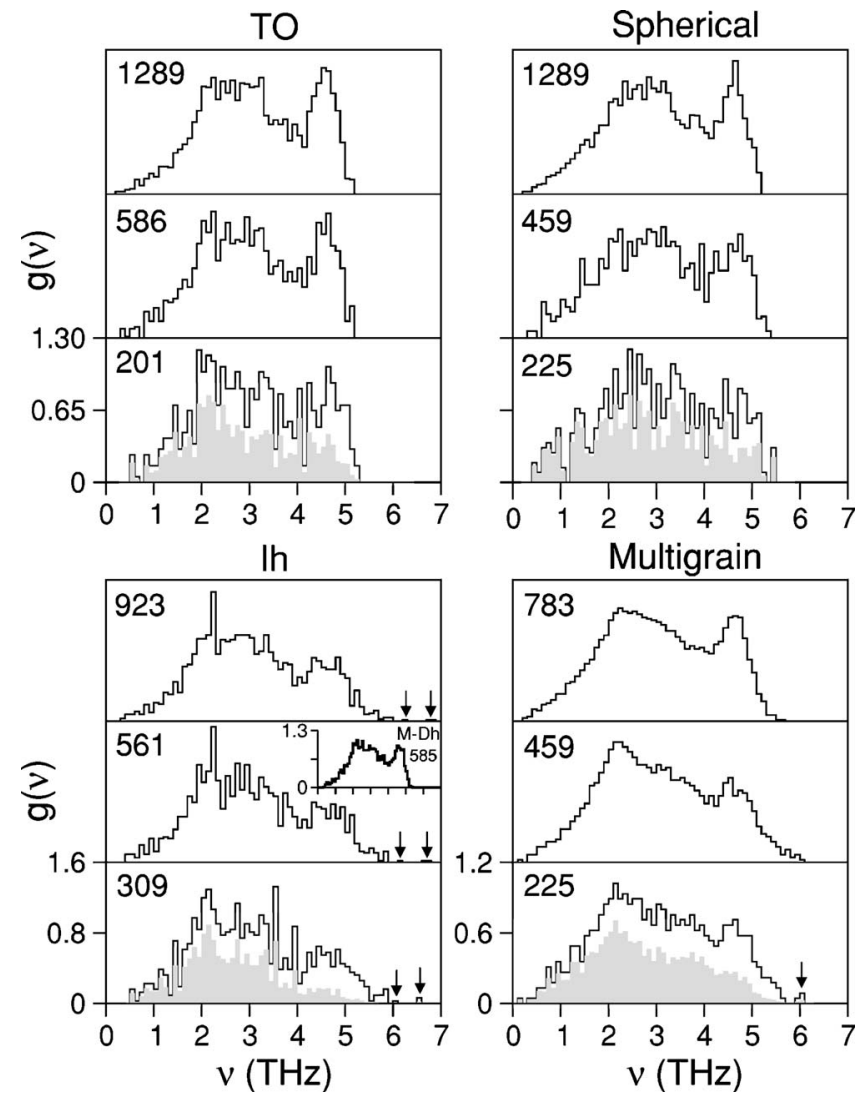

FIG. 6. Morphology and size effects on vibrational density of states $g(\nu)$. Shaded histograms show the partial density of states due to surface atoms. Bin size is $0.1 \mathrm{THz}$. At low and high frequencies, $g(\nu)$ depends significantly on geometrical details of the nanograins. Density of states at $\nu<2 \mathrm{THz}$ is higher in spherical grains than in TO. For faceted and Ih grains, the longitudinal-phonon (LP) peaks around $4.7 \mathrm{THz}$ have nearly the same amplitude regardless of size. In contrast, LP's amplitude grows with grain size in multigrain and spherical grains. Grain boundaries in multigrain and twined Ih lead to a frequency tail beyond the maximum value in single-crystalline grains. No tail is seen in Mark's decahedra, whereas the LP peak is visible. [See inset in panel Ih, 561 for $g(\nu)$ of a $585 \mathrm{M}$-Dh.] Arrows indicate features arising from phonons strongly localized in the center of the grains.

shown). More important, Mark's decahedra do not show a high-frequency tail in DOS. Multigrain and spherical nanograins: In spherical grains, protrusions increase $g(\nu)$ below 2 and above $5 \mathrm{THz}$ relative to faceted grains. Although less prominently than in multigrains, the amplitude of LP peaks also increases and narrows with size. In multigrain, (i) the amplitude of the LP peaks grows and narrows with size. Remarkably, Pasquini et al. ${ }^{21}$ observed a similar build-up of longitudinal phonons of nanocrystalline $\alpha$-Fe. ${ }^{22}$ (ii) Similarly to $\mathrm{Ih}$, a frequency tail appears at high frequencies. (An arrow points to features arising from inner atoms.)

Finally, let us mention that Derlet and Swygenhoven ${ }^{23}$ have recently shown that the features at higher frequencies in the DOS of nanocomposites originate from vibrations strongly localized in grain boundaries. The frequency tail that appears in $g(\nu)$ for Ih and multigrain is an intrinsic feature of nanoscopic metals with grain boundaries. This re- 


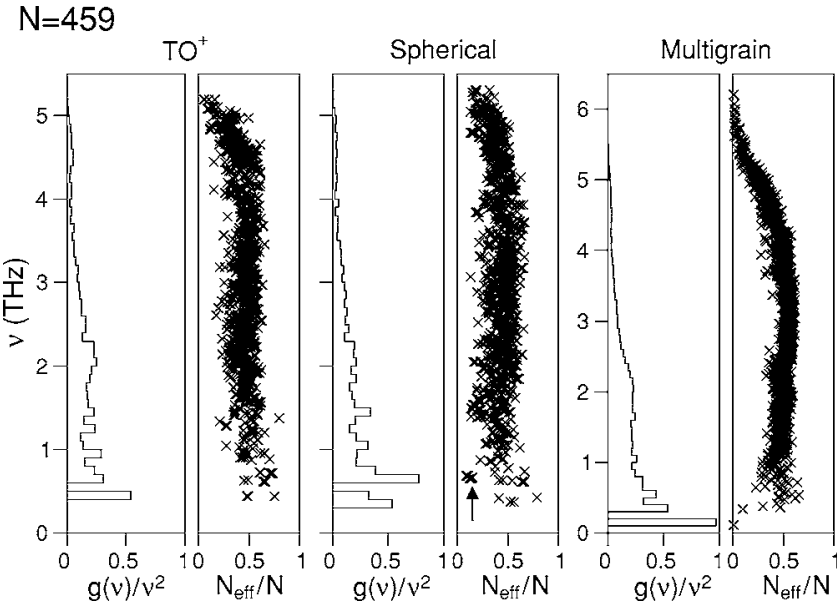

FIG. 7. Morphology effects on the reduced density of states $g(\nu) / \nu^{2}$ and the effective number of atoms $N_{\text {eff }}(\nu)$ in a phonon of frequency $\nu$. For wavelike modes, $N_{\text {eff }} / N=1 / 2$. Only multigrain shows a $\nu^{2}$ distribution from 1 to slightly over $2 \mathrm{THz}$. In all grains, edges and vertices significantly enhance $g(\nu) / \nu^{2}$ below $1 \mathrm{THz}$. An arrow indicates modes heavily localized on the protrusions of the spherical grain, which coincide with the peak around $0.6 \mathrm{THz}$. In the multigrain, grain boundaries and irregular surfaces are responsible for the reduced fluctuations shown in $N_{\text {eff }} / N$.

markable finding reveals the strong connection between extended nanocrystalline materials and finite size nanoscopic grains.

\section{Phonon localization}

The participation ratio $N_{\text {eff }}(\nu)$ (see the Appendix) indicates the effective number of atoms participating in a phonon with frequency $\nu$. Thereby, $N_{\text {eff }}(\nu)$ measures the degree of phonon localization. In a sample with $N$ atoms, $N_{\text {eff }} / N$ $\simeq 1 / 2$ for extended, wavelike phonon modes. ${ }^{24}$ Note that when we identify a phonon mode as being localized $\left(N_{\text {eff }} / N \ll 1 / 2\right)$ we do not imply necessarily that the amplitude of the atomic vibrations decays away exponentially from a particular atomic site, as it is the case for Andersonlocalized vibrational states. ${ }^{25}$ In addition, while Anderson localization takes place in disordered systems, the term localization in the context of our results is broader as grains have a surface and, therefore, phonon localization-i.e., phonon modes with a small number of atoms involved in the vibration-takes place even in crystalline grains.

Figure 7 shows $N_{\text {eff }}(\nu)$ scaled by $N$, and the reduced vibrational density of states $g(\nu) / \nu^{2}$ for $N=459 \mathrm{TO}^{+}$, spherical and multigrain. The central results can be summarized as follows. (a) General features of $N_{\text {eff }}$ representative for all nanograins: (i) High-frequency phonons become more localized in both single-crystalline and multigrain specimens. In the latter, grain boundaries lead to a higher degree of localization. This is similar to what occurs in nanocrystalline materials; ${ }^{23}$ a result that shows that nanograins capture some of the physics of their massive, nanocrystalline counterparts. $^{26}$ (ii) At low frequencies, the more localized the vibrations the higher the enhancement of DOS. In particular,
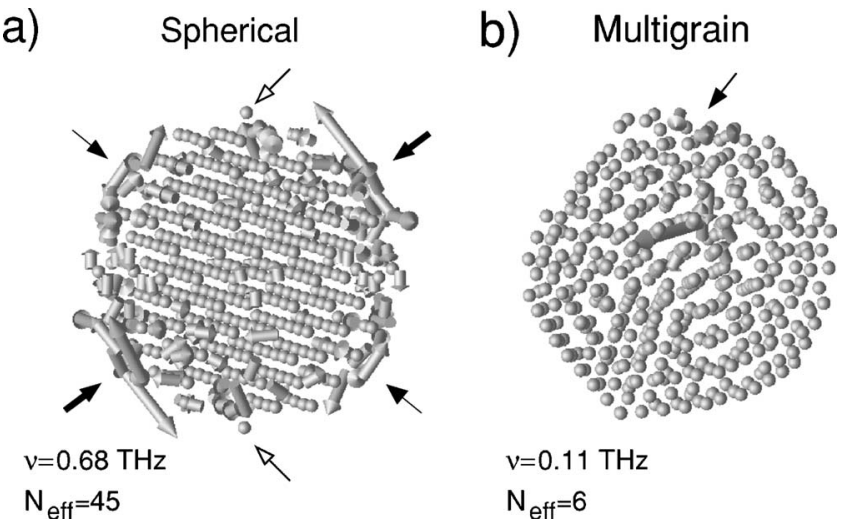

FIG. 8. Displacement vectors for two selected low-frequency, localized phonon modes in $N=459$ spherical (a) and multigrain (b) samples. For each mode, we also show the frequency $(\nu)$ and $N_{\text {eff }}$ (Sec. III C and Appendix). In (a), the atoms with significant displacement-vector amplitude are those located on the six-atom protrusions (indicated with solid arrows) that decorate the [111] facets of the underlying TO grain [Fig. 1(c)]. Single-atom protrusions that decorate the [001] facets have negligible displacementvector amplitude (open arrow). In (b), the phonon mode involves a very low number of atoms $\left(N_{\text {eff }}=6\right)$ that are around a vertex vacancy (indicated with an arrow) and grain boundaries.

spherical grains show a peak (arrow, Fig. 7) around $0.6 \mathrm{THz}$ due to localized phonon modes at the surface protrusions. In turn, multigrain shows a similar enhancement due to phonons localized around vertex vacancies and in atoms in the vicinity of grain boundaries. In particular, two lowfrequency modes at $0.1 \mathrm{THz}$ and $0.3 \mathrm{THz}$ that involve $\sim 5$ and 50 atoms, respectively, are responsible for the lowfrequency peak in $g(\nu) / \nu^{2}$. Faceted single-crystal and twined samples (not shown) show a lesser enhancement, arising only from the edges and vertices. (iii) $N_{\text {eff }}$ significantly fluctuates around $N / 2$ for single-crystal samples, twined Ih and $\mathrm{M}-\mathrm{Dh}$; whereas in multigrain, irregular shape, surface disorder, and defects reduce these fluctuations.

(b) Two prominent features appear in $g(\nu) / \nu^{2}$ : (i) The nearly quadratic behavior of $g(\nu)$ from 1-2.2 THz for multigrain. The frequency extent of this Debye-type $\left[g(\nu) \sim \nu^{2}\right]$ region depends on the nature of disorder and defectsmultiple grain boundaries, stacking faults, and vertex vacancies. Such Debye dependence has been observed in iron nanocomposites by Pasquini and co-workers. ${ }^{21}$ The Debye constant derived from the quadratic interval is nearly twice as big as the bulk value for Ag. Remarkably, such enhancement of the Debye constant has also been observed by Fultz and co-workers in nanocrystalline iron. ${ }^{27}$ It should be noted that while we have found Debye-type behavior in a few nanograins of different sizes, the statistics we have is limited due to the intrinsic difficulty in generating competitive (Sec. II) multigrain samples using long molecular-dynamics simulations. (ii) The significant correlation between $N_{\text {eff }}(\nu)$ and $g(\nu) / \nu^{2}$, which shows that the degree of phonon localization in nanoscopic metal grains can be derived from a measurement of their vibrational density of states, after dividing by $\nu^{2}$.

Finally, Figs. 8(a) and 8(b) show, respectively, displace- 
ment vectors for two low-frequency, localized $\left(N_{\text {eff }} / N\right.$ $\ll 1 / 2)$ phonon modes in $N=459$ spherical and multigrain samples. These phonon modes are among those responsible for the low-frequency peaks in $g(\nu) / \nu^{2}$ (Fig. 7). We find the following. (i) In the spherical grain, the phonon mode is one of a threefold degenerate set with frequency $\nu=0.68 \mathrm{THz}$ and $N_{\text {eff }} / N=0.098$. This mode is mostly localized in the sixatom protrusions that decorate the hexagonal, [111] facets of the underlying TO grain (Fig. 1 and Sec. II). The amplitude of the displacement vectors of the atoms in the protrusions is different depending on which facet the protrusion is located, as indicated by solid, thick- and thin-stem arrows in Fig. 8(a), whereas displacement vectors of atoms in diametrically opposed protrusions have the same amplitude but different phase. Note that in this phonon mode [Fig. 8(a)] the singleatom protrusions located on top of [001] facets have negligible displacement-vector amplitude (open arrows). (ii) The phonon mode in the multigrain sample [Fig. 8(b)] is the lowest allowed phonon. Its frequency is $\nu=0.11 \mathrm{THz}$ and $N_{\text {eff }} / N=0.013$. The displacement vectors with significant amplitude locate around a vertex vacancy, indicated with an arrow, and along grain boundaries.

\section{SUMMARY}

To understand in detail the vibrational properties of nanoscopic metal grains we performed atomistic simulations of the phonon spectra-frequencies and displacement vectors-of silver grains with realistic morphologies. Our results show that morphology introduces a high degree of complexity into the phonon spectra, total and partial vibrational density of states, and phonon localization. The most prominent features are the following. (a) Low-energy, singlecrystalline grains present nearly pure torsional and radial phonon modes. The grains' sharp edges and atomic lattice split off the acoustic gap quintet predicted by ET into a doublet and triplet, with a magnitude that depends on the relative number of atoms at the boundary of different facets. When compared to faceted grains of the same size, high-energy, spherical models that present regular protrusions on the surface have a smaller acoustic gap and a higher total DOS at frequencies $\nu<2 \mathrm{THz}$. (b) Twined icosahedra also have a breathing mode. Strain in these grains leads to a higher acoustic gap, and a high-frequency tail in the DOS that originates from core atoms. This tail extends beyond the highest frequency in single-crystalline grains. Remarkably, neither twined and strained Mark's decahedra have a breathing mode nor do they exhibit a high-frequency tail on the DOS. (c) Nanograins with grain boundaries and surface disorder do not have degenerate frequencies and the acoustic gap is significantly reduced. These nanograins are the only ones that exhibit low-frequency $\nu^{2}$ DOS in the interval 1-2 THz. The extent of this region depends on the nature of disorder.
These predictions, while illustrated here for silver, should be valid for other nanoscopic metal grains. Our simulations show the complexity of the vibrational properties of metal nanograins, and point out similarities between the vibrational density of states of metal nanograins with grain boundaries and surface disorder and that of massive nanocrystalline samples. Finally, this work is a necessary step toward understanding the size-dependent electron-phonon coupling in nanoscopic metals.

\section{ACKNOWLEDGMENTS}

Funds from U. S. DOE Grant No. DE-FG02-99ER45795 and computational resources from the National Center of Supercomputer Applications (NCSA) made this research possible. National Science Foundation Grant No. NSF-DMR03-25939-ITR supports the Materials Computation Center. The authors thank Professor Jian-Min Zuo (UIUC) for fruitful discussions. This research began at the Materials Computation Center (MCC). One of the authors (G.A.N.) acknowledges its hospitality, and the opportunity to participate in the MCC Visitor Program.

\section{APPENDIX: METHODS}

Nanograin's structure and energetics: The literature contains extensive studies regarding the energetics of metal clusters. In this work, we consider known geometries and perform total energy relaxations in $\mathrm{Ag}$ nanograins. We also perform extensive simulated annealing to get complex, partially disordered morphologies. ${ }^{28}$ To calculate the total energy $\mathcal{E}$ we employ the Daw and Baskes embedded atom method (EAM). ${ }^{29}$ We use the Voter and Chen parametrization of the Ag potential.

Vibrational properties: Once we generate stable nanograin morphologies we calculate phonon frequencies $\nu$ and displacement vectors $\left\{\vec{u}_{\nu}^{i}\right\}$-where $i$ labels an atom located at equilibrium position $\vec{R}^{i}$ in the grain-by direct diagonalization of the dynamical matrix $D_{\alpha \beta}^{i j}=\partial^{2} \mathcal{E} / \partial R_{\alpha}^{i} \partial R_{\beta}^{j}$. $R_{\alpha}^{i}$ is a Cartesian component $(\alpha, \beta=x, y, z)$ of $\vec{R}^{i}$. Note that we consider free-standing nanograins, hence, the first six phonons correspond to rigid-body translations and rotations along and about the principal axis, respectively. These frequencies do not enter in our analysis.

The vibrational density of states (DOS) $g(\nu)=\sum_{j=1}^{N} g_{j}(\nu)$, where $g_{j}(\nu) \Delta \nu=(1 / N) \Sigma_{\nu} n(\nu) \sqrt{M}\left|\vec{u}_{\nu}^{j}\right|$ is the partial (local) vibrational density of states at atomic site $j ; \vec{u}_{\nu}^{j}$ is the displacement vector of atom $j ; n(\nu)$ is the number of phonons between $\nu$ and $\nu+\Delta \nu$; and $M$ is the mass of a $\mathrm{Ag}$ atom.

The equality $N_{\text {eff }}(\nu)=M_{1}^{2} / M_{2}$ defines the participation ratio. ${ }^{30-32}$ Here, $M_{p}=\Sigma_{i}^{N}\left[\varepsilon_{i}(\nu)\right]^{p}$ are momenta of the mean kinetic energy $\varepsilon_{i}(\nu)$ of atom $i$. By using the calculated displacement vectors of atom $i$ in a phonon with frequency $\nu$ one readily calculates $\varepsilon_{i}(\nu)$. 
*Present address: National Renewable Energy Laboratory, Golden, Colorado 80401.

${ }^{1}$ A. Arbouet, C. Voisin, D. Christofilos, P. Langot, N. Del Fatti, F. Vallée, J. Lermé, G. Celep, E. Cottancin, M. Gaudry, M. Pellarin, M. Broyer, M. Maillard, M. P. Pileni, and M. Treguer, Phys. Rev. Lett. 90, 177401 (2003).

${ }^{2}$ J. Hodak, A. Henglein, and G. V. Hartland, J. Chem. Phys. 112, 5942 (2000).

${ }^{3}$ B. A. Smith, J. Z. Zhang, U. Giebel, and G. Schmid, Chem. Phys. Lett. 270, 139 (1997).

${ }^{4}$ C. L. Cleveland, W. D. Luedtke, and Uzi Landman, Phys. Rev. B 60, 5065 (1999); C. L. Cleveland, U. Landman, T. G. Schaaff, M. N. Shafigullin, P. W. Stephens, and R. L. Whetten, Phys. Rev. Lett. 79, 1873 (1997); C. L. Cleveland, U. Landman, M. N. Shafigullin, P. W. Stephens, and R. L. Whetten, Z. Phys. D: At., Mol. Clusters 40, 503 (1997).

${ }^{5}$ F. Baletto, R. Ferrando, A. Fortunelli, E. Montalenti, and C. Mottet, J. Chem. Phys. 116, 3856 (2002).

${ }^{6}$ F. Baletto, C. Mottet, and R. Ferrando, Chem. Phys. Lett. 354, 82 (2002); Phys. Rev. B 63, 155408 (2001).

${ }^{7}$ F. Baletto and R. Ferrando, Rev. Mod. Phys. 77, 371 (2005).

${ }^{8}$ A. Kara and T. S. Rahman, Phys. Rev. Lett. 81, 1453 (1998).

${ }^{9}$ D. Y. Sun, X. G. Gong, and X.-Q. Wang, Phys. Rev. B 63, 193412 (2001).

${ }^{10}$ R. Meyer, L. J. Lewis, S. Prakash, and P. Entel, Phys. Rev. B 68, 104303 (2003).

${ }^{11}$ One may argue that such grains would be energetically unfavorable; however, these are more competitive than the simple models; see Sec. II.

${ }^{12}$ L. D. Landau and E. M. Lifshitz, Theory of Elasticity (Pergamon New York, 1975).

${ }^{13}$ K. R. Patton and M. R. Geller, Phys. Rev. B 67, 155418 (2003).

${ }^{14}$ A. Tamura, K. Higeta, and T. Ichinokawa, J. Phys. C 15, 4975 (1982).

${ }^{15}$ Variants of Ih are those that include vertex and on-center vacancies. As first shown in C. Mottet, G. Tréglia, and B. Legrand, Surf. Sci. 383, L719 (1997), our simulations also predict that the cohesive energy of on-center-vacant icosahedra improves slightly over Ih; as the vacancy helps to relieve the accumulated strain. The cohesive energy does not improve by adding vertex vacancies in $\mathrm{Ih}$.

${ }^{16}$ C. Y. Yang, J. Cryst. Growth 47, 274 (1979), and references therein.

${ }^{17}$ L. D. Marks, Rep. Prog. Phys. 57, 603 (1994), and references therein.
${ }^{18}$ Here, sphericity means how oblate the grain is. A comparison of the momenta of inertia along principal axes of a grain and a sphere measures the actual degree of sphericity. See K. Manninen, J. Akola, and M. Manninen, Phys. Rev. B 68, 235412 (2003) where the authors correlate the momenta of inertia with the total energy of ultrasmall $(N<110)$ Al nanograins.

${ }^{19}$ O. L. Anderson, Physical Acoustics-Principles and Methods, edited by W. P. Mason (Academic, New York, 1965), Vol. III, Part B.

${ }^{20}$ Other choices of bin size give similar results.

${ }^{21}$ L. Pasquini, A. Barla, A. I. Chumakov, O. Leupold, R. Rüffer, A. Deriu, and E. Bonetti, Phys. Rev. B 66, 073410 (2002).

${ }^{22}$ Note that $\alpha$-Fe nanocrystalline samples used in the experiments of Ref. 21 are not free-standing nanograins. They consist of several nanocrystals of nearly the same size that are prepared from a powder. The typical size of the nanocrystals depends on the synthesis and varies from nearly $6-13 \mathrm{~nm}$.

${ }^{23}$ P. M. Derlet and H. V. Swygenhoven, Phys. Rev. Lett. 92, 035505 (2004); P. M. Derlet, R. Meyer, L. J. Lewis, U. Stuhr, and H. Van Swygenhoven, ibid. 87, 205501 (2000).

${ }^{24}$ For example, in a linear harmonic chain $N_{\text {eff }} / N=1 / 2$ at all frequencies.

${ }^{25}$ J. D. Maynard, Rev. Mod. Phys. 73, 401 (2001).

${ }^{26}$ The similarity between the DOS of nanoscopic grains and massive, nanocrystalline specimens depends on considering a range of grain's morphologies, in contrast to Meyer et al. (Ref. 10) who only compared spherical grains and a model nanocomposite.

${ }^{27}$ B. Fultz, C. C. Ahn, E. E. Alp, W. Sturhahn, and T. S. Toellner, Phys. Rev. Lett. 79, 937 (1997).

${ }^{28}$ Our simulated annealing is based on molecular dynamics. We use Verlet's algorithm with time steps from $1-2$ fs, and run multimillion-step simulations in the microcanonical ensemble. Typically, we cool down a molten droplet down to 300-500 K at rates from $20-50 \mathrm{~K} / \mathrm{ns}$. Then, we relax the atomic positions to minimize $\mathcal{E}$. Although we use high cooling rates, they are practical and fall within the range normally used in the literature.

${ }^{29}$ M. S. Daw and M. I. Baskes, Phys. Rev. B 29, 6443 (1984); Phys. Rev. Lett. 50, 1285 (1983).

${ }^{30}$ W. Jin, P. Vashishta, R. K. Kalia, and J. P. Rino, Phys. Rev. B 48, 9359 (1993).

${ }^{31}$ S. R. Nagel, G. S. Grest, and A. Rahman, Phys. Rev. Lett. 53, 368 (1984).

${ }^{32}$ P. T. Dean, Rev. Mod. Phys. 44, 127 (1972). 